\title{
On the index of Simon's congruence for piecewise testability
}

\author{
P. Karandikara,b,1,2, M. Kufleitner ${ }^{\mathrm{c}, 3}$, Ph. Schnoebelen ${ }^{\mathrm{a}, 2}$ \\ ${ }^{a}$ Lab. Specification 85 Verification, CNRS UMR 864383 ENS Cachan, France \\ ${ }^{b}$ Chennai Mathematical Institute, Chennai, India \\ ${ }^{c}$ Institut für Formale Methoden der Informatik, University of Stuttgart, Germany
}

\begin{abstract}
Simon's congruence, denoted $\sim_{n}$, relates words having the same subwords of length up to $n$. We show that, over a "k-letter alphabet, the number of words modulo $\sim_{n}$ is in $2^{\Theta\left(n^{k-1} \log n\right)}$.
\end{abstract}

Keywords: Combinatorics of words; Piecewise testable languages; Subwords and subsequences.

\section{'1. Introduction}

Piecewise testable languages, introduced by Imre Simon 'in the 1970s, are a family of star-free regular languages 'that are definable by the presence and absence of given '(scattered) subwords [1, 2, 3]. Formally, a language $L \subseteq$ ' $A *$ is $n$-piecewise testable if $x \in L$ and $x \sim_{n} y$ imply $y \in L$, "where $x \sim_{n} y \stackrel{\text { def }}{\Leftrightarrow} x$ and $y$ have the same subwords of length at most $n$ (see next section for all definitions missing in this 'introduction). Piecewise testable languages are important because they are the languages defined by $\mathcal{B} \Sigma_{1}$ formulae, a simple fragment of first-order logic that is prominent in database queries [4]. They also occur in learning theory [5], 'computational linguistics [6], etc.

It is easy to see that $\sim_{n}$ is a congruence with finite index 'and Sakarovitch and Simon raised the question of how to better characterize or evaluate this number [2, p. 110]. Let 'us write $C_{k}(n)$ for the number of $\sim_{n}$ classes over $k$ letters, i.e., when $|A|=k$. It is clear that $C_{k}(n) \geq k^{n}$ since two "words $x, y \in A \leq n$ (i.e., of length at most $n$ ) are related by $\sim_{n}$ only if they are equal. In fact, this reasoning gives

$$
C_{k}(n) \geq k^{n}+k^{n-1}+\cdots+k+1=\frac{k^{n+1}-1}{k-1}
$$

(assuming $k \neq 1$ ). On the other hand, any congruence class in $\sim_{n}$ is completely characterized by a set of subwords in $A \leq n$, hence

$$
C_{k}(n) \leq 2^{\frac{k^{n+1}-1}{k-1}}
$$

Estimating the size of $C_{k}(n)$ has applications in descriptive complexity, for example for estimating the number of $n$ piecewise testable languages (over a given alphabet), or for bounding the size of canonical automata for $n$-piecewise testable languages [7, 8, 9].

\footnotetext{
${ }^{1}$ Partially supported by Tata Consultancy Services.

${ }^{2}$ Supported by ANR grant 11-BS02-001-01.

${ }^{3}$ Supported by DFG grant DI 435/5-2.
}

Unfortunately the above bounds, summarized as $k^{n} \leq$ $C_{k}(n) \leq 2^{k^{n+1}}$, leave a large ("exponential") gap and it is not clear towards which side is the actual value leaning 4 Eq. (11) gives a lower bound that is obviously very naive since it only counts the simplest classes. On the other hand, Eq. (2) too makes wide simplifications since not every subset of $A^{\leq n}$ corresponds to a congruence class. For example, if aa and $\mathrm{bb}$ are subwords of some $x$ then necessarily $x$ also has ab or ba among its length 2 subwords.

Since the question of estimating $C_{k}(n)$ was raised in [2] (and to the best of our knowledge) no progress has been made on the question, until Kátai-Urbán et al. proved the following bounds:

Theorem 1.1 (Kátai-Urbán et al. [10]). For all $k>$ 1 ,

$$
\begin{aligned}
\frac{k^{n}}{3^{2}} \log k & \leq \log C_{k}(n)<3^{n} k^{n} \log k & & \text { if } n \text { is even, } \\
\frac{k^{n}}{3^{n^{2}}} & <\log C_{k}(n)<3^{n} k^{n} & & \text { if } n \text { is odd. }
\end{aligned}
$$

The proof is based on two reductions, one showing $C_{k+\ell}(n+2) \geq C_{k}^{\ell+2}(n)$ for proving lower bounds, and one showing $C_{k}(n+2) \leq(k+1)^{2 k} C_{k}^{2 k-1}(n)$ for proving upper bounds. For fixed $n$, Theorem 1.1 allows to estimate the asymptotic value of $\log C_{k}(n)$ as a function of $k$ : it is in $\Theta\left(k^{n}\right)$ or $\Theta\left(k^{n} \log k\right)$ depending on the parity of $n$. However, these bounds do not say how, for fixed $k, C_{k}(n)$ grows as a function of $n$, which is a more natural question in settings where the alphabet is fixed, and where $n$ comes from, e.g., the number of variables in a $\mathcal{B} \Sigma_{1}$ formula. In particular, the lower bound is useless for $n \geq k$ since in this case $k^{n} / 3^{n^{2}}<1$.

\footnotetext{
${ }^{4}$ Comparing the bounds from Eqs. (1) and (2) with actual values does not bring much light here since the magnitude of $C_{k}(n)$ makes it hard to compute beyond some very small values of $k$ and $n$, see Table B.1
} 
Our contribution. In this article, we provide the following bounds:

Theorem 1.2. For all $k, n>1$,

$$
\begin{aligned}
\left(\frac{n}{k}\right)^{k-1} \log _{2}\left(\frac{n}{k}\right) & <\log _{2} C_{k}(n) \\
& <k\left(\frac{n+2 k-3}{k-1}\right)^{k-1} \log _{2} n \log _{2} k .
\end{aligned}
$$

Thus, for fixed $k, \log C_{k}(n)$ is in $\Theta\left(n^{k-1} \log n\right)$. Compared with Theorem 1.1 our bounds are much tighter for fixed $k$ (and much wider for fixed $n$ ).

The proof of Theorem 1.2 relies on two new reductions that allows us to relate $C_{k}(n)$ with $C_{k-1}$ instead of relating it with $C_{k}(n-2)$ as in [10]. The article is organized as follows. Section 2 recalls the necessary notations and definitions; the lower bound is proved in Section 3 while the upper bound is proved in Section 4. An appendix lists the exact values of $C_{k}(n)$ for small $n$ and $k$ that we managed to compute.

\section{Basics}

We consider words $x, y, w, \ldots$ over a finite $k$-letter alphabet $A_{k}=\left\{\mathrm{a}_{1}, \ldots, \mathrm{a}_{k}\right\}$ sometimes written more simply $A=\{\mathrm{a}, \mathrm{b}, \ldots\}$. The empty word is denoted $\epsilon$, concatenation is denoted multiplicatively. Given a word $x \in A^{*}$ and a letter a $\in A$, we write $|x|$ and $|x|_{\mathrm{a}}$ for, respectively, the length of $x$, and the number of occurrences of a in $x$.

We write $x \preccurlyeq y$ to denote that a word $x$ is a subsequence of $y$, also called a (scattered) subword. Formally, $x \preccurlyeq y$ iff $x=x_{1} \cdots x_{\ell}$ and there are words $y_{0}, y_{1}, \ldots, y_{\ell}$ such that $y=y_{0} x_{1} y_{1} \cdots x_{\ell} y_{\ell}$. It is well-known that $\preccurlyeq$ is a partial ordering and a monoid precongruence.

For any $n \in \mathbb{N}$, we write $x \sim_{n} y$ when $x$ and $y$ have the same subwords of length $\leq n$. For example $x \stackrel{\text { def }}{=} \mathrm{abacb} \sim \sim_{2} \quad y \stackrel{\text { def }}{=}$ baaacbb since both words have $\{\epsilon, \mathrm{a}, \mathrm{b}, \mathrm{c}, \mathrm{aa}, \mathrm{ab}, \mathrm{ac}, \mathrm{ba}, \mathrm{bb}, \mathrm{bc}, \mathrm{cb}\}$ as subwords of length $\leq 2$. However $x \chi_{3} y$ since $x \succcurlyeq$ aba $\npreceq y$. Note that $\sim_{0} \supseteq \sim_{1} \supseteq \sim_{2} \supseteq \cdots$, and that $x \sim_{0} y$ holds trivially. It is well-known (and easy to see) that each $\sim_{n}$ is a congruence since the subwords of some $x y$ are the concatenations of a subword of $x$ and a subword of $y$. Simon defined a piecewise testable language as any $L \subseteq A^{*}$ that is closed by $\sim_{n}$ for some $n$ [1]. These are exactly the languages definable by $\mathcal{B} \Sigma_{1}(<, \mathrm{a}, \mathrm{b}, \ldots)$ formulae [4], i.e., by Boolean combinations of existential first-order formulae with monadic predicates of the form a $(i)$, stating that the $i$-th letter of a word is a. For example, $L=A^{*} \mathrm{a} A^{*} \mathrm{~b} A^{*}=\left\{x \in A^{*} \mid \mathrm{ab} \preccurlyeq x\right\}$ is definable with the following $\Sigma_{1}$ formula:

$$
\exists i: \exists j: i<j \wedge \mathrm{a}(i) \wedge \mathrm{b}(j) .
$$

The index of $\sim_{n}$. Since there are only finitely many words of length $\leq n$, the congruence $\sim_{n}$ partitions $A_{k}^{*}$ in finitely many classes, and we write $C_{k}(n)$ for the number of such classes, i.e., the cardinal of $A_{k}^{*} / \sim_{n}$.
The following is easy to see:

$$
C_{1}(n)=n+1, \quad C_{k}(0)=1, \quad C_{k}(1)=2^{k} .
$$

Indeed, for words over a single letter a, $x \sim_{n} y$ iff $|x|=$ $|y|<n$ or $|x| \geq n \leq|y|$, hence the first equality. The second equality restates that $\sim_{0}$ is trivial, as noted above. For the third equality, one notes that $x \sim_{1} y$ if, and only if, the same set of letters is occurring in $x$ and $y$, and that there are $2^{k}$ such sets of occurring letters.

\section{Lower bound}

The first half of Theorem [1.2 is proved by first establishing a combinatorial inequality on the $C_{k}(n)$ 's (Proposition [3.3) and then using it to derive Proposition 3.4 .

Consider two words $x, y \in A^{*}$ and a letter $a \in A$.

Lemma 3.1. If $x \sim_{n} y$, then $\min \left(|x|_{a}, n\right)=\min \left(|y|_{a}, n\right)$.

Proof (Sketch). If $|x|_{a}=p<n$ then $a^{p} \preccurlyeq x \neq a^{p+1}$. From $x \sim_{n} y$ we deduce $a^{p} \preccurlyeq y \nsucc a^{p+1}$, hence $|y|_{a}=p$.

Fix now $k \geq 2$, let $A=A_{k}=\left\{\mathrm{a}_{1}, \ldots, \mathrm{a}_{k}\right\}$ and assume $x \sim_{n} y$. If $|x|_{\mathrm{a}_{k}}=p<n$, then $x$ is some $x_{0} \mathrm{a}_{k} x_{1} \cdots \mathrm{a}_{k} x_{p}$ with $x_{i} \in A_{k-1}^{*}$ for $i=0, \ldots, p$. By Lemma 3.1, $y$ too is some $y_{0} \mathrm{a}_{k} y_{1} \cdots \mathrm{a}_{k} y_{p}$ with $y_{i} \in A_{k-1}^{*}$.

Lemma 3.2. $x_{i} \sim_{n-p} y_{i}$ for all $i=0, \ldots, p$.

Proof. Suppose $w \preccurlyeq x_{i}$ and $|w| \leq n-p$. Let $w^{\prime} \stackrel{\text { def }}{=}$ $\mathrm{a}_{k}^{i} w \mathrm{a}_{k}^{p-i}$. Clearly $w^{\prime} \preccurlyeq x$ and thus $w^{\prime} \preccurlyeq y$ since $x \sim_{n} y$ and $\left|w^{\prime}\right| \leq n$. Now $w^{\prime}=\mathrm{a}_{k}^{i} w \mathrm{a}_{k}^{p-i} \preccurlyeq y$ entails $w \preccurlyeq y_{i}$.

With a symmetric reasoning we show that every subword of $y_{i}$ having length $\leq n-p$ is a subword of $x_{i}$ and we conclude $x_{i} \sim_{n-p} y_{i}$.

Proposition 3.3. For $k \geq 2, C_{k}(n) \geq \sum_{p=0}^{n} C_{k-1}^{p+1}(n-p)$.

Proof. For words $x=x_{0} \mathrm{a}_{k} x_{1} \ldots x_{p-1} \mathrm{a}_{k} x_{p}$ with exactly $p<n$ occurrences of $\mathrm{a}_{k}$, we have $C_{k-1}(n-p)$ possible choices of $\sim_{n-p}$ equivalence classes for each $x_{i}(i=$ $0, \ldots, p)$. By Lemma 3.2 all such choices will result in $\chi_{n}$ words, hence there are exactly $C_{k-1}^{p+1}(n-p)$ classes of words with $p<n$ occurrences of $\mathrm{a}_{k}$. By Lemma 3.1, these classes are disjoint for different values of $p$, hence we can add the $C_{k-1}^{p+1}(n-p)$ 's. There remain words with $p \geq n$ occurrences of $\mathrm{a}_{k}$, accounting for at least 1 , i.e., $C_{k-1}^{n+1}(0)$, additional class.

Proposition 3.4. For all $k, n>0$ :

$$
\log _{2} C_{k}(n)>\left(\frac{n}{k}\right)^{k-1} \log _{2}\left(\frac{n}{k}\right)
$$


Proof. Eq. (44) holds trivially when $\log _{2}\left(\frac{n}{k}\right) \leq 0$. Hence there only remains to consider the cases where $n>k$. We reason by induction on $k$. For $k=1$, Eq. (3) gives $\log _{2} C_{1}(n)=\log _{2}(n+1)>\log _{2} n=\left(\frac{n}{1}\right)^{0} \log _{2}\left(\frac{n}{1}\right)$. For the inductive case, Proposition 3.3 yields $C_{k+1}(n) \geq C_{k}^{p+1}(n-$ $p$ ) for all $p \in\{0, \ldots, n\}$. For $p=\left\lfloor\frac{n}{k+1}\right\rfloor$ this yields

$$
\begin{aligned}
\log _{2} C_{k+1}(n) & \geq(p+1) \log _{2} C_{k}(n-p) \\
& >(p+1)\left(\frac{n-p}{k}\right)^{k-1} \log _{2}\left(\frac{n-p}{k}\right)
\end{aligned}
$$

by ind. hyp., noting that $n-p>0$,

$$
\geq \frac{n}{k+1}\left(\frac{n}{k+1}\right)^{k-1} \log _{2}\left(\frac{n}{k+1}\right)
$$

since $\frac{n-p}{k} \geq \frac{n}{k+1} \geq 1$,

$$
=\left(\frac{n}{k+1}\right)^{k} \log _{2}\left(\frac{n}{k+1}\right)
$$

as desired.

\section{Upper bound}

The second half of Theorem 1.2 is again by establishing a combinatorial inequality on the $C_{k}(n)$ 's (Proposition 4.3) and then using it to derive Proposition 4.4.

Fix $k>0$ and consider words in $A_{k}^{*}$. We say that a word $x$ is rich if all the $k$ letters of $A_{k}$ occur in it, and that it is poor otherwise. For $\ell>0$, we further say that $x$ is $\ell$-rich if it can be written as a concatenation of $\ell$ rich factors (by extension " $x$ is 0 -rich" means that $x$ is poor). The richness of $x$ is the largest $\ell \in \mathbb{N}$ such that $x$ is $\ell$-rich. Note that $\forall a \in A_{k}:|x|_{a} \geq \ell$ does not imply that $x$ is $\ell$-rich. We shall use the following easy result:

Lemma 4.1. If $x_{1}$ and $x_{2}$ are respectively $\ell_{1}$-rich and $\ell_{2}$ rich, then $y \sim_{n} y^{\prime}$ implies $x_{1} y x_{2} \sim_{\ell_{1}+n+\ell_{2}} x_{1} y^{\prime} x_{2}$.

Proof. A subword $u$ of $x_{1} y x_{2}$ can be decomposed as $u=$ $u_{1} v u_{2}$ where $u_{1}$ is the largest prefix of $u$ that is a subword of $x$ and $u_{2}$ is the largest suffix of the remaining $u_{1}^{-1} u$ that is a subword of $x_{2}$. Thus $v \preccurlyeq y$ since $u \preccurlyeq x_{1} y x_{2}$. Now, since $x_{1}$ is $\ell_{1}$-rich, $\left|u_{1}\right| \geq \ell_{1}$ (unless $u$ is too short), and similarly $\left|u_{2}\right| \geq \ell_{2}$ (unless ...). Finally $|v| \leq n$ when $|u| \leq \ell_{1}+n+\ell_{2}$, and then $v \preccurlyeq y^{\prime}$ since $y \sim_{n} y^{\prime}$, entailing $u \preccurlyeq x_{1} y^{\prime} x_{2}$. A symmetrical reasoning shows that subwords of $x_{1} y^{\prime} x_{2}$ of length $\leq \ell_{1}+n+\ell_{2}$ are subwords of $x_{1} y x_{2}$ and we are done.

The rich factorization of $x \in A_{k}^{*}$ is the decomposition $x=x_{1} a_{1} \cdots x_{m} a_{m} y$ obtained in the following way: if $x$ is poor, we let $m=0$ and $y=x$; otherwise $x$ is rich, we let $x_{1} a_{1}$ (with $a_{1} \in A_{k}$ ) be the shortest prefix of $x$ that is rich, write $x=x_{1} a_{1} x^{\prime}$ and let $x_{2} a_{2} \ldots x_{m} a_{m} y$ be the rich factorization of the remaining suffix $x^{\prime}$. By construction $m$ is the richness of $x$. E.g., assuming $k=3$, the following is a rich factorization with $m=2$ :

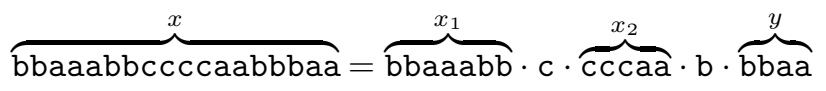

Note that, by definition, $x_{1}, \ldots, x_{m}$ and $y$ are poor.

Lemma 4.2. Consider two words $x, x^{\prime}$ of richness $m$ and with rich factorizations $x=x_{1} a_{1} \ldots x_{m} a_{m} y$ and $x^{\prime}=$ $x_{1}^{\prime} a_{1} \ldots x_{m}^{\prime} a_{m} y^{\prime}$. Suppose that $y \sim_{n} y^{\prime}$ and that $x_{i} \sim_{n+1}$ $x_{i}^{\prime}$ for all $i=1, \ldots, m$. Then $x \sim_{n+m} x^{\prime}$.

Proof. By repeatedly using Lemma 4.1, one shows

$$
\begin{aligned}
& x_{1} a_{1} x_{2} a_{2} \ldots x_{m} a_{m} y \sim_{n+m} x_{1}^{\prime} a_{1} x_{2} a_{2} \ldots x_{m} a_{m} y \\
& \sim_{n+m} x_{1}^{\prime} a_{1} x_{2}^{\prime} a_{2} \ldots x_{m} a_{m} y \\
& \vdots \\
& \\
& \sim_{n+m} x_{1}^{\prime} a_{1} x_{2}^{\prime} a_{2} \ldots x_{m}^{\prime} a_{m} y \\
& \sim_{n+m} x_{1}^{\prime} a_{1} x_{2}^{\prime} a_{2} \ldots x_{m}^{\prime} a_{m} y^{\prime}
\end{aligned}
$$

using the fact that each factor $x_{i} a_{i}$ is rich.

Proposition 4.3. For all $n \geq 0$ and $k \geq 2$,

$$
C_{k}(n) \leq 1+\sum_{m=0}^{n-1} k^{m+1} C_{k-1}^{m}(n-m+1) C_{k-1}(n-m) .
$$

Furthermore, for $k=2$,

$$
C_{2}(n) \leq 2 \sum_{m=0}^{2 n-1} n^{m}=2 \frac{n^{2 n}-1}{n-1}
$$

Proof. Consider two words $x, x^{\prime}$ and their rich factorization $x=x_{1} a_{1} \ldots x_{m} a_{m} y$ and $x^{\prime}=x_{1}^{\prime} a_{1}^{\prime} \ldots x_{\ell}^{\prime} a_{\ell}^{\prime} y^{\prime}$. By Lemma 4.2 they belong to the same $\sim_{n}$ class if $\ell=m$, $y \sim_{n-m} y^{\prime}$, and $a_{i}=a_{i}^{\prime}$ and $x_{i} \sim_{n-m+1} x_{i}^{\prime}$ for all $i=1, \ldots, m$. Now for every fixed $m$, there are at most $k^{m}$ choices for the $a_{i}$ 's, $C_{k-1}^{m}(n-m+1)$ non-equivalent choices for the $x_{i}$ 's, $k C_{k-1}(n-m)$ choices for $y$ and a letter that is missing in it. We only need to consider $m$ varying up to $n-1$ since all words of richness $\geq n$ are $\sim_{n}$-equivalent, accounting for one additional possible $\sim_{n}$ class.

For the second inequality, assume that $k=2$ and $A_{2}=$ $\{\mathrm{a}, \mathrm{b}\}$. A word $x \in A_{2}^{*}$ can be decomposed as a sequence of $m$ non-empty blocks of the same letter, of the form, e.g., $x=\mathrm{a}^{\ell_{1}} \mathrm{~b}^{\ell_{2}} \mathrm{a}^{\ell_{3}} \mathrm{~b}^{\ell_{4}} \ldots \mathrm{a}^{\ell_{m}}$ (this example assumes that $x$ starts and ends with a, hence $m$ is odd). If two words like $x=\mathrm{a}^{\ell_{1}} \mathrm{~b}^{\ell_{2}} \mathrm{a}^{\ell_{3}} \mathrm{~b}^{\ell_{4}} \ldots \mathrm{a}^{\ell_{m}}$ and $x^{\prime}=\mathrm{a}^{\ell_{1}^{\prime}} \mathrm{b}^{\ell_{2}^{\prime}} \mathrm{a}^{\ell_{3}^{\prime}} \mathrm{b}^{\ell_{4}^{\prime}} \ldots \mathrm{a}^{\ell_{m}^{\prime}}$ have the same first letter a, the same alternation depth $m$, and have $\min \left(\ell_{i}, n\right)=\min \left(\ell_{i}^{\prime}, n\right)$ for all $i=1, \ldots, m$, then they are $\sim_{n}$-equivalent. For a given $m>0$, there are 2 possibilities for choosing the first letter and $n^{m}$ non-equivalent choices for the $\ell_{i}$ 's. Finally, all words with alternation depths $m \geq 2 n$ are $\sim_{n}$-equivalent, hence we can restrict our attention to $1 \leq m \leq 2 n-1$. The extra summand $2 n^{0}$ in Eq. (5) accounts for the single class with $m \geq 2 n$ and the single class with $m=0$. 
Proposition 4.4. For all $k, n>1$ :

$$
C_{k}(n)<2^{k\left(\frac{n+2 k-3}{k-1}\right)^{k-1} \log _{2} n \log _{2} k} .
$$

Proof. By induction on $k$. For $k=2$, Eq. (5) yields:

$$
C_{2}(n) \leq 2 \frac{n^{2 n}-1}{n-1}<n \frac{n^{2 n+1}}{1}
$$

since $n \geq 2$,

$$
\begin{aligned}
& =n^{2 n+2}=2^{2(n+1) \log _{2} n} \\
& =2^{k\left(\frac{n+2 k-3}{k-1}\right)^{k-1} \log _{2} n \log _{2} k} .
\end{aligned}
$$

For the inductive case, Proposition 4.3 yields:

$$
\begin{aligned}
C_{k+1}(n) \leq & 1+\sum_{m=0}^{n-1}(k+1)^{m+1} C_{k}^{m}(n-m+1) C_{k}(n-m) \\
= & 1+(k+1) C_{k}(n) \\
& +\sum_{m=1}^{n-1}(k+1)^{m+1} C_{k}^{m}(n-m+1) C_{k}(n-m) \\
< & (k+1)^{n} C_{k}(n)+\sum_{m=1}^{n-1}(k+1)^{n} C_{k}^{m+1}(n-m+1)
\end{aligned}
$$

since $C_{k}(q) \leq C_{k}(q+1)$,

$$
\begin{aligned}
< & (k+1)^{n} 2^{k\left(\frac{n+2 k-3}{k-1}\right)^{k-1} \log _{2} n \log _{2} k} \\
& +\sum_{m=1}^{n-1}(k+1)^{n} 2^{k(m+1)\left(\frac{n-m+2 k-2}{k-1}\right)^{k-1} \log _{2} n \log _{2} k}
\end{aligned}
$$

by ind. hyp.,

$$
<(k+1)^{n} \sum_{m=0}^{n-1} 2^{k(m+1)\left(\frac{n-m+2 k-2}{k-1}\right)^{k-1} \log _{2} n \log _{2} k} .
$$

Since $(m+1)\left(\frac{n-m+2 k-2}{k-1}\right)^{k-1} \leq\left(\frac{n+2 k-1}{k}\right)^{k}$ for all $m \in$ $\{0, \ldots, n-1\} \rightarrow$ see Appendix A , we may proceed with:

$$
\begin{aligned}
C_{k+1}(n) & <(k+1)^{n} \sum_{m=0}^{n-1} 2^{k\left(\frac{n+2 k-1}{k}\right)^{k} \log _{2} n \log _{2} k} \\
& =n(k+1)^{n} 2^{k\left(\frac{n+2 k-1}{k}\right)^{k} \log _{2} n \log _{2} k} \\
& =2^{\log _{2} n+n \log _{2}(k+1)+k\left(\frac{n+2 k-1}{k}\right)^{k} \log _{2} n \log _{2} k} \\
& <2^{\left(\log _{2} n+n+k\left(\frac{n+2 k-1}{k}\right)^{k} \log _{2} n\right) \log _{2}(k+1)} \\
& <2^{(k+1)\left(\frac{n+2 k-1}{k}\right)^{k} \log _{2} n \log _{2}(k+1)}
\end{aligned}
$$

since $\log _{2} n+n<\left(\frac{n+2 k-1}{k}\right)^{k} \log _{2} n$ (see below). This is the desired bound.

To see that $\log _{2} n+n<\left(\frac{n+2 k-1}{k}\right)^{k} \log _{2} n$, we use

$$
\begin{aligned}
\left(\frac{n+2 k-1}{k}\right)^{k} & >\left(\frac{n}{k}+1\right)^{k}=\sum_{j=0}^{k}\left(\begin{array}{l}
k \\
j
\end{array}\right) \cdot\left(\frac{n}{k}\right)^{j} \\
& =1+k \cdot\left(\frac{n}{k}\right)+\cdots \geq n+1
\end{aligned}
$$

This completes the proof.
By combining the two bounds in Propositions 3.4 and 4.4 we obtain Theorem 1.2 implying that $\log C_{k}(n)$ is in $\Theta\left(n^{k-1} \log n\right)$ for fixed alphabet size $k$.

\section{Conclusion}

We proved that, over a fixed $k$-letter alphabet, $C_{k}(n)$ is in $2^{\Theta\left(n^{k-1} \log n\right)}$. This shows that $C_{k}(n)$ is not doubly exponential in $n$ as Eq. (2) and Theorem 1.1 would allow. It also is not simply exponential, bounded by a term of the form $2^{f(k) \cdot n^{c}}$ where the exponent $c$ does not depend on $k$.

We are still far from having a precise understanding of how $C_{k}(n)$ behaves and there are obvious directions for improving Theorem 1.2. For example, its bounds are not monotonic in $k$ (while the bounds in Theorem 1.1 are not monotonic in $n$ ) and it only partially uses the combinatorial inequalities given by Propositions 3.3 and 4.3 .

Acknowledgments. We thank J. Berstel, J.-É. Pin and M. Zeitoun for their comments and suggestions.

\section{References}

[1] I. Simon, Piecewise testable events, in: Proc. 2nd GI Conf. on Automata Theory and Formal Languages, volume 33 of Lecture Notes in Computer Science, Springer, 1975, pp. 214-222. doi 10.1007/3-540-07407-4_23

[2] J. Sakarovitch, I. Simon, Subwords, in: M. Lothaire (Ed.), Combinatorics on words, volume 17 of Encyclopedia of Mathematics and Its Applications, Cambridge Univ. Press, 1983, pp. 105-142.

[3] J.-E. Pin, Varieties of Formal Languages, Plenum, New-York, 1986.

[4] V. Diekert, P. Gastin, M. Kufleitner, A survey on small fragments of first-order logic over finite words, Int. J. Foundations of Computer Science 19 (2008) 513-548.

[5] L. Kontorovich, C. Cortes, M. Mohri, Kernel methods for learning languages, Theoretical Computer Science 405 (2008) 223236.

[6] J. Rogers, J. Heinz, G. Bailey, M. Edlefsen, M. Visscher, D. Wellcome, S. Wibel, On languages piecewise testable in the strict sense, in: Proc. 10th and 11th Biennal Conf. Mathematics of Language (MOL 10), volume 6149 of Lecture Notes in Computer Science, Springer, 2010, pp. 255-265. doi $10.1007 / 978-3-642-14322-9 \_19$

[7] W. Czerwiński, W. Martens, T. Masopust, Efficient separability of regular languages by subsequences and suffixes, in: Proc. 40th Int. Coll. Automata, Languages, and Programming (ICALP 2013), volume 7966 of Lecture Notes in Computer Science, Springer, 2013, pp. 150-161. doi 10.1007/978-3-642-39212-2_16

[8] O. Klíma, L. Polák, Alternative automata characterization of piecewise testable languages, in: Proc. 17th Int. Conf. Developments in Language Theory (DLT 2013), volume 7907 of Lecture Notes in Computer Science, Springer, 2013, pp. 289-300. doi 10.1007/978-3-642-38771-5_26

[9] Th. Place, L. van Rooijen, M. Zeitoun, Separating regular languages by piecewise testable and unambiguous languages, in: Proc. 38th Int. Symp. Math. Found. Comp. Sci. (MFCS 2013), volume 8087 of Lecture Notes in Computer Science, Springer, 2013, pp. 729-740. doi 10.1007/978-3-642-40313-2_64

[10] K. Kátai-Urbán, P. P. Pach, G. Pluhár, A. Pongrácz, C. Szabó, On the word problem for syntactic monoids of piecewise testable languages, Semigroup Forum 84 (2012) 323-332. 


\section{Appendix A. Additional proofs}

We prove that $(m+1)\left(\frac{n-m+2 k-2}{k-1}\right)^{k-1} \leq\left(\frac{n+2 k-1}{k}\right)^{k}$ for all $m=0, \ldots, n-1$, an inequality that was used to establish Proposition 4.4

For $k>0$ and $x, y \in \mathbb{R}$, let

$$
\begin{aligned}
F_{k}(x) & \stackrel{\text { def }}{=}\left(\frac{x+2 k-1}{k}\right)^{k}, \\
G_{k, x}(y) & \stackrel{\text { def }}{=}(y+1) F_{k}(x-y+1)=\frac{(y+1)(x-y+2 k)^{k}}{k^{k}} .
\end{aligned}
$$

Let us check that $G_{k, x}\left(\frac{k+x}{k+1}\right)=F_{k+1}(x)$ for any $k>0$ and $x \geq 0$ :

$$
\begin{aligned}
G_{k, x}\left(\frac{k+x}{k+1}\right) & =\left(\frac{k+x}{k+1}+1\right) \frac{1}{k^{k}}\left(x-\frac{k+x}{k+1}+2 k\right)^{k} \\
& =\frac{x+2 k+1}{k+1} \frac{1}{k^{k}}\left(\frac{k x+2 k^{2}+k}{k+1}\right)^{k} \\
& =\frac{x+2 k+1}{k+1} \frac{1}{k^{k}}\left(\frac{k}{k+1}\right)^{k}(x+2 k+1)^{k} \\
& =\left(\frac{x+2 k+1}{k+1}\right)^{k+1}=F_{k+1}(x) .
\end{aligned}
$$

We now claim that $G_{k, x}(y) \leq F_{k+1}(x)$ for all $y \in[0, x]$. For $n, k \geq 2$, the claim entails $G_{k-1, n}(m) \leq F_{k}(m)$, i.e. $(m+1)\left(\frac{n-m+2 k-2}{k-1}\right)^{k-1} \leq\left(\frac{n+2 k-1}{k}\right)^{k}$, for $m=0, \ldots, n-1$ as announced.

ProOF (OF THE ClAIM). Let $y_{\max } \stackrel{\text { def }}{=} \frac{k+x}{k+1}$. We prove that $G_{k, x}(y) \leq G_{k, x}\left(y_{\max }\right)$ and conclude using Eq. $(\dagger)$ : $G_{k, x}$ is well-defined and differentiable over $\mathbb{R}$, its derivative is

$$
\begin{aligned}
G_{k, x}^{\prime}(y) & =\frac{(x-y+2 k)^{k}-(y+1) k(x-y+2 k)^{k-1}}{k^{k}} \\
& =\frac{(x-y+2 k)^{k-1}}{k^{k}}((x-y+2 k)-(y+1) k) \\
& =\frac{(x-y+2 k)^{k-1}}{k^{k}}(x+k-y(k+1)) .
\end{aligned}
$$

Thus $G_{k, x}^{\prime}(y)$ is 0 for $y=y_{\max }$, is strictly positive for $0 \leq y<y_{\max }$, and strictly negative for $y_{\max }<y \leq x$. Hence, over $[0, x], G_{k, x}$ reaches its maximum at $y_{\max }$.

\section{Appendix B. First values for $C_{k}(n)$}

We computed the first values of $C_{k}(n)$ by a brute-force method that listed all minimal representatives of $\sim_{n}$ equivalence classes over a $k$-letter alphabet. Here $x$ is minimal if $x \sim_{n} y$ implies $\left(|x|<|y|\right.$ or $\left(|x|=|y|\right.$ and $\left.\left.x \leq_{\operatorname{lex}} y\right)\right)$. Every equivalence class has a unique minimal representative. Note that if a concatenation $x x^{\prime}$ is minimal then both $x$ and $x^{\prime}$ are. Therefore, when listing the minimal representatives in order of increasing length, it is possible to stop when, for some length $\ell$, one finds no minimal representatives. In that case we know that there cannot exist minimal representatives of length $>\ell$.

The cells left blank in the table were not computed for lack of memory. 


\begin{tabular}{|c|c|c|c|c|c|c|c|c|c|}
\hline & $k=1$ & $k=2$ & $k=3$ & $k=4$ & $k=5$ & $k=6$ & $k=7$ & $k=8$ & $k$ \\
\hline$n=0$ & 1 & 1 & 1 & 1 & 1 & 1 & 1 & 1 & 1 \\
\hline$n=1$ & 2 & 4 & 8 & 16 & 32 & 64 & 128 & 256 & $2^{k}$ \\
\hline$n=2$ & 3 & 16 & 152 & 2326 & 52132 & 1602420 & 64529264 & $\geq 173 \cdot 10^{7}$ & \\
\hline$n=3$ & 4 & 68 & 5312 & 1395588 & 1031153002 & $\geq 23 \cdot 10^{7}$ & & & \\
\hline$n=4$ & 5 & 312 & 334202 & $\geq 73 \cdot 10^{7}$ & & & & & \\
\hline$n=5$ & 6 & 1560 & 38450477 & & & & & & \\
\hline$n=6$ & 7 & 8528 & $\geq 39 \cdot 10^{7}$ & & & & & & \\
\hline$n=7$ & 8 & 50864 & & & & & & & \\
\hline$n=8$ & 9 & 329248 & & & & & & & \\
\hline$n=9$ & 10 & 2298592 & & & & & & & \\
\hline$n=10$ & 11 & 17203264 & & & & & & & \\
\hline$n=11$ & 12 & 137289920 & & & & & & & \\
\hline$n$ & $n+1$ & & & & & & & & \\
\hline
\end{tabular}

